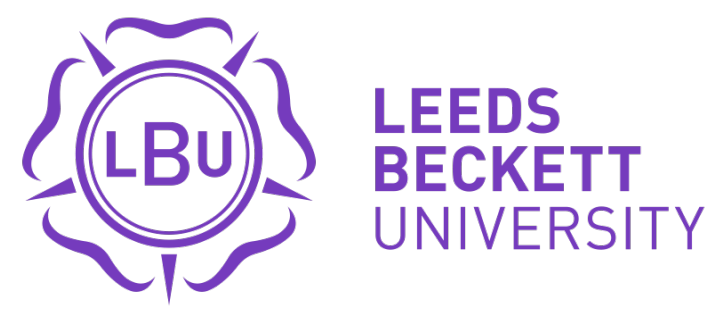

Citation:

Naimi, A and Deng, J and Shimjith, SR and Arul, J (2021) Dynamic Neural Network-based Feedback Linearization Control of a Pressurized Water Reactor. 2020 13th International Conference on Developments in eSystems Engineering (DeSE), 13. pp. 228-232. ISSN 2161-1351 DOI: https://doi.org/10.1109/DeSE51703.2020.9450737

Link to Leeds Beckett Repository record:

https://eprints.leedsbeckett.ac.uk/id/eprint/7936/

Document Version:

Article (Accepted Version)

(C) 2021 IEEE. Personal use of this material is permitted. Permission from IEEE must be obtained for all other uses, in any current or future media, including reprinting/republishing this material for advertising or promotional purposes, creating new collective works, for resale or redistribution to servers or lists, or reuse of any copyrighted component of this work in other works.

The aim of the Leeds Beckett Repository is to provide open access to our research, as required by funder policies and permitted by publishers and copyright law.

The Leeds Beckett repository holds a wide range of publications, each of which has been checked for copyright and the relevant embargo period has been applied by the Research Services team.

We operate on a standard take-down policy. If you are the author or publisher of an output and you would like it removed from the repository, please contact us and we will investigate on a case-by-case basis.

Each thesis in the repository has been cleared where necessary by the author for third party copyright. If you would like a thesis to be removed from the repository or believe there is an issue with copyright, please contact us on openaccess@leedsbeckett.ac.uk and we will investigate on a case-by-case basis. 


\title{
Dynamic Neural Network-based Feedback Linearization Control for a Pressurized Water Reactor
}

\author{
Amine Naimi, Jiamei Deng, S. R. Shimjith, A. John Arul
}

\begin{abstract}
This note presents a nonlinear control approach using dynamic neural network (DNN)-based feedback linearization (FBL) for nuclear reactor power control. The reactor model adopted in this study is based on neutronic dynamic and thermal-hydraulic models. The nonlinear plant is identified by a single-layer DNN trained using Quasi-Newton and InteriorPoint methods. The feedback linearization scheme is combined with a Proportional-Integral (P-I) controller and simulations show good performance of the proposed controller. The efficacy of the controller is evaluated in the load-following mode of operation. Moreover, the fault-tolerance performance of the proposed approach is tested.
\end{abstract}

Index Terms - Feedback Linearization, Dynamic Neural Network, Nonlinear Control System, Pressurized Water Reactor, Nuclear Power Plant.

\section{INTRODUCTION}

Nuclear power plants (NPPs) are complex systems, and the implementation of desirable control for the core is necessary to ensure safety and effectiveness of the plant. In order to guarantee efficiency and maintain safe operation, the plant needs to achieve stability under uncertainties caused by unmodeled dynamics, parameter variations and linearization.

Proportional-integral-derivative (PID) controllers have still a predominant role in various industries including nuclear applications [1]. Hence, several PID gains tuning methods were developed [2], and various methods were employed to optimize these gains for load-following issues in nuclear plants. For instance, Mousakazemi et al. [3] proposed a PID controller for power-level control of a pressurized water reactor (PWR), the controller gains were optimised using the particle swarm optimization (PSO) algorithm.

Linear control approaches are subject to lacking robustness which is required to ensure good operational performance of the NPPs. Therefore, in recent years, various nonlinear control techniques have been used for nuclear reactor power control, such as neural networks methods [4], fuzzy logic methods [5], intelligent control systems [6] and robust optimal control systems [7]. The above nonlinear control methods require exact mathematical model of the PWR reactor system.

A few researchers applied feedback linearization (FBL) methods to control nuclear reactors, such as for the control

Amine Naimi (amine.naimi01@gmail.com) and Jiamei Deng (j.deng@leedsbeckett.ac.uk) are with School of Built Environment, Engineering, and Computing, Leeds Beckett University, Leeds, United Kingdom.

S.R. Shimjith (srshim@barc.gov.in) is with Reactor Control System Design Section, Bhabha Atomic Research Centre, Homi Bhabha National, Mumbai, India.

John Arul (arul@barc.gov.in) is with Reactor Shielding and Data Division, Indira Gandhi Centre for Atomic Research, Kalpakkam, India. of the power-level for the PWRs which was discussed in [8] Eom et al. [9] presented a robust disturbance observer-based FBL control system of a research reactor which possesses the ability of estimating states as well as limiting the core power change rate. Ansarifar et al. [10] proposed a combination of Conventional FBL and Sliding mode control for Axial-Offset control of pressurized water reactors during load following operation. In [11], a Dynamic neural network (DNN) was proposed to estimate a PWR reactor and was dedicated to being used with FBL approach.

FBL techniques with artificial intelligence have been an active research in the last 3 decades [12]. For instance, a multi-layer controller-based was proposed to ensure bounded control actions [13], moreover, a neuro-controller using FBL was studied in [14]. Practical applications of DNN-Based FBL control can be found in different areas. For example, it was applied to an electromechanical process [15] and few applications can be reported in the automotive field [16], [17]]. Deng et al. [18] demonstrated the benefits of using this approach by integrating it into a model predictive control scheme. However, FBL and DNN methods have not been applied to NPP control.

In this paper, an FBL approach based on a single-layer DNN is used for the control of a PWR. The FBL is combined with a linear PID controller to ensure the achievement of a stable control. The paper is arranged as follows: Section II introduces the PWR model. Section III presents the DNN-based system identification. Section IV implements the proposed controller. Section V shows the simulation results. Finally, section VI concludes this work.

\section{NON-LINEAR PWR MOdeL}

In this paper, the mathematical model of the PWR assumes point kinetics equation coupled with six delayed neutron groups and lumped thermal hydraulic model. For control purpose, the reactivity and the power are considered as input and output of the reactor, respectively. The PWR model used can be found in the literature [19]. The dynamic model is described as follows:

$$
\begin{aligned}
\frac{d P}{d t} & =\frac{\rho_{t}-\sum_{i=1}^{6} \beta_{i}}{\Lambda} P+\sum_{i=1}^{6} \lambda_{i} C_{i}, \\
\frac{d C_{i}}{d t} & =\frac{\beta_{i}}{\Lambda} P-\lambda_{i} C_{i}, \quad i=1,2, \ldots 6 .
\end{aligned}
$$




$$
\begin{aligned}
\frac{d T_{f}}{d t} & =H_{f} P_{n}-\frac{1}{\tau_{f}}\left(T_{f}-T_{c 1}\right), \\
\frac{d T_{c 1}}{d t} & =H_{c} P_{n}+\frac{1}{\tau_{c}}\left(T_{f}-T_{c 1}\right)-\frac{2}{\tau_{r}}\left(T_{c 1}-T_{c i n}\right), \\
\frac{d T_{c 2}}{d t} & =H_{c} P_{n}+\frac{1}{\tau_{c}}\left(T_{f}-T_{c 1}\right)-\frac{2}{\tau_{r}}\left(T_{c 2}-T_{c 1}\right) .
\end{aligned}
$$

In the above set of equations, $\mathrm{P}$ is the neutronic power, $\Lambda$ is the prompt neutron; $C_{i}, \Lambda$ and $\beta_{i}$ are delayed neutron precursors's concentration, decay constant and fraction of delayed neutrons, respectively. $T_{f}, T_{c 1}$, and $T_{c 2}$ are the temperatures at fuel, coolant node 1 and node 2, respectively. $H_{f}$ and $H_{c}$ are proportionality constants. $\tau_{f}, \tau_{c}$, and $\tau_{r}$ are time constants. The effects of variation in temperatures of fuel and coolants are considered in terms of reactivity feedback. Hence, the total reactivity is given by:

$$
\begin{aligned}
& \rho_{t}=\rho_{\text {rod }}+\rho_{f}+\rho_{c 1}+\rho_{c 2} \\
& \rho_{t}=\rho_{\text {rod }}+\alpha_{f} T_{f}+\alpha_{c}\left(T_{c 1}+T_{c 2}\right)
\end{aligned}
$$

where $\rho_{\text {rod }}, \rho_{f}, \rho_{c 1}$, and $\rho_{c 2}$ denote the reactivity due to control rod, fuel temperature, coolant temperature at node 1 and 2, respectively. $\alpha_{f}$ and $\alpha_{c}$ denote the temperature coefficients of reactivity due to fuel and coolant, respectively.

\section{DNN-BASED SYSTEM IDENTIFICATION}

DNNs have, in comparison to static neural networks, more complex architectures. They provide the possibility to analyze time-dependent data, since the network elements are made of interconnected dynamic neurons. Such networks can be represented by a nonlinear state space model. The general equation of the DNN can be expressed as follows [12]:

$$
\begin{aligned}
& \dot{x}(t)=f(x(t), u(t), \theta) \\
& \hat{y}(t)=h(x(t), \theta)
\end{aligned}
$$

where $x \in \mathbb{R}^{N}$ are the states of the network, $u \in \mathbb{R}^{m}$, is the external input, $\theta$ is a vector of parameters of the network, and $y \in \mathbb{R}^{p}$ is the output. $f$ and $h$ are functions that represent the structure of the network and the relationships between the output and state, respectively. The structure of the DNN used in this paper us a specific case of (7) and can be expressed as the equation (8):

$$
\dot{x}_{i}=-\beta_{i} x_{i}+\sum_{j=1}^{N} \omega_{i j} \sigma\left(x_{j}\right)+\sum_{j=1}^{m} \gamma_{i j} u_{j}
$$

where $\beta_{i}, \omega_{i}$, and $\gamma_{i}$ are adjustable weights, $\frac{1}{\beta_{i}}$ is a positive time constant, $x_{i}$ the states of the system and $u_{j}$ the input signals. The block diagram of a dynamic neuron is shown in Fig. 1. The vectorized form of (8) is given by:

$$
\begin{aligned}
& \dot{x}=-\beta x+\omega \sigma(x)+\gamma u \\
& \hat{y}=C x
\end{aligned}
$$

where $x$ are coordinates on $\mathbb{R}^{N}, \omega \in \mathbb{R}^{N \times N}, \sigma(x)=$ $\left[\sigma\left(x_{1}\right) \cdots \sigma\left(x_{N}\right)\right]^{T}, \gamma \in \mathbb{R}^{N \times m}, u \in \mathbb{R}^{m}, C=$ $\left[I_{p \times p} 0_{p \times(N-p)}\right]$, and $\beta \in \mathbb{R}^{N \times N}$ is a diagnosable matrix

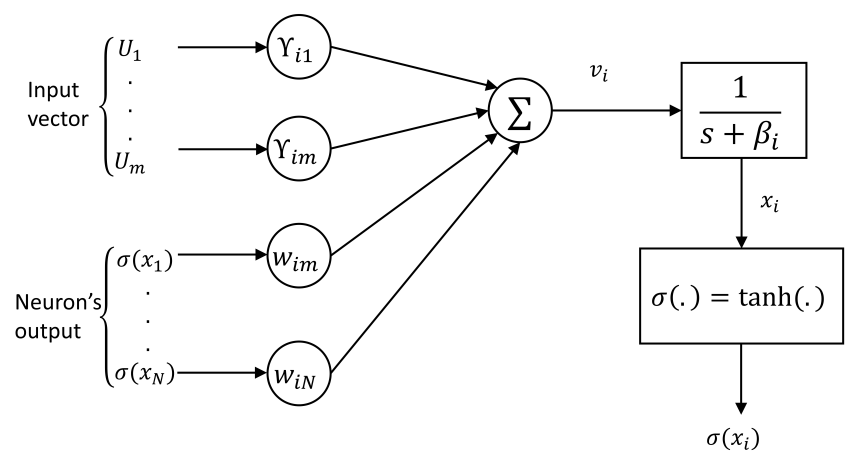

Fig. 1: A dynamic neuron.

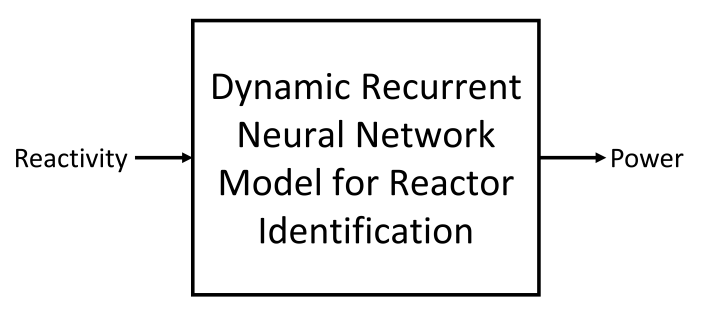

Fig. 2: Reactor Identifier block diagram.

with diagonal elements $\left\{\beta_{1} \cdots \beta_{N}\right\}$.

In an earlier work [11], the DNN described previously was trained to learn the dynamic of the process from reactivity and power data sets (Fig.2). Trainings were carried out using Quasi-Newton algorithm (Q-N) as well as InteriorPoint methods. The model found was a second order for the two DNNs, more details about the approach can be found in [11]. The DNN model trained using Q-N methods can be represented as follows:

$$
\begin{aligned}
\dot{x}_{1} & =-\beta_{1} x_{1}+\omega_{11} \sigma\left(x_{1}\right)+\omega_{12} \sigma\left(x_{2}\right)+\gamma_{1} u \\
\dot{x}_{2} & =-\beta_{2} x_{2}+\omega_{21} \sigma\left(x_{1}\right)+\omega_{22} \sigma\left(x_{2}\right)+\gamma_{2} u \\
y & =C x
\end{aligned}
$$

where

$$
\begin{aligned}
& \beta=\left[\begin{array}{cc}
0.5767 & 0 \\
0 & -0.1038
\end{array}\right] ; \omega=\left[\begin{array}{cc}
-0.4523 & 0.7762 \\
-0.1850 & -0.3670
\end{array}\right] \\
& \gamma=\left[\begin{array}{ll}
0.620 & 0.1837
\end{array}\right]^{T} ; C=[10] .
\end{aligned}
$$

\section{Controller Design}

FBL is a common approach used for the control of nonlinear systems. The main objective of this control is to transform a nonlinear dynamic system into a linear system using nonlinear coordinate transformations and nonlinear state feedback. By eliminating nonlinearities in the closed loop system, conventional linear control techniques can be applied. FBL may be applied to nonlinear systems of the 
following form:

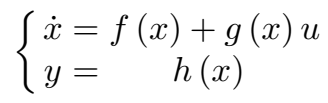

where $x$ is a state vector, $u$ is a control function and $y$ is a controlled output. Also, the system described in (15) has a relative degree if [12]:

$$
\left\{\begin{array}{c}
L_{g} L_{f}^{k} h(x)=0, \\
L_{g} L_{f}^{r-1} h(x) \neq 0
\end{array}\right.
$$

where $L_{p} h(x)=\partial h(x) / \partial x$. $p$ for $p=f, g$ is the lie derivative of the function $f(x)$.

According to this definition of relative degree and considering the DNN model described in (12-14), the relative degree of the reactor system is 1 . Hence, the desirable control law according to feedback-linearization can be represented as follows:

$$
u=P(x)+Q(x) v
$$

where

$$
\begin{array}{r}
P(x)=-A(x)^{-1}+B(x), \\
Q(x)=A(x)^{-1}
\end{array}
$$

with

$$
A(x)=\left[\begin{array}{ccc}
\hat{\lambda}_{1 r 1} L_{g 1} L_{f}^{r 1-1} h(x) & \ldots & \hat{\lambda}_{1 r 1} L_{g p} L_{f}^{r 1-1} h_{1}(x) \\
\ldots & \ldots & \ldots \\
\hat{\lambda}_{p r p} L_{g 1} L_{f}^{r p-1} h_{p}(x) & \ldots & \hat{\lambda}_{p r p} L_{g p} L_{f}^{r p-1} h_{p}(x)
\end{array}\right]_{p \times p}
$$$$
B(x)=\left[\begin{array}{c}
\sum_{k=0}^{r 1} \hat{\lambda}_{1 k} L_{f}^{k} h_{1}(x) \\
\ldots \\
\sum_{k=0}^{r p} \hat{\lambda}_{p k} L_{f}^{k} h_{p}(x)
\end{array}\right]_{p \times 1}
$$

$u=\frac{1}{\lambda_{1} \gamma_{1}}\left(v-\lambda_{0} x_{1}-\lambda_{1}\left(\beta_{1} x_{1}+\omega_{11} \sigma\left(x_{1}\right)+\omega_{12} \sigma(x 62)\right)\right.$

where $\hat{\lambda}_{1 k}$ are arbitrary values. $\lambda_{0}$ and $\lambda_{1}$ were chosen such that the linearized system $\mathrm{v}-\mathrm{y}$ is subject to the same static gain and time constant as the DNN model given in equations (12-14). In this study, a conventional P-I controller is used in the outer control loop. The proposed control strategy is described in Fig.3.

\section{Simulation Results}

To evaluate the performance of the proposed controller, a load-following transient is considered. The transient is chosen to be within the range $50 \%<\mathrm{P}<100 \%$ as it is where the controller is performing well. The desired core power level is changing as follows: $100 \% \rightarrow 50 \% \rightarrow 65 \% \rightarrow 80 \% \rightarrow 95 \% \rightarrow 80 \% \rightarrow 65 \% \rightarrow 50 \%$.

The performance of the controller is shown in Fig. 4. It can be seen that the controller tracks well the load changes. The

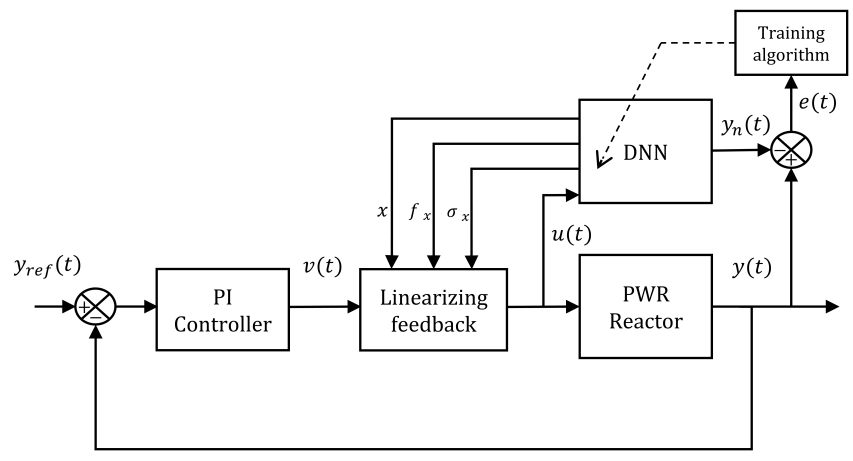

Fig. 3: Structure of the control strategy.

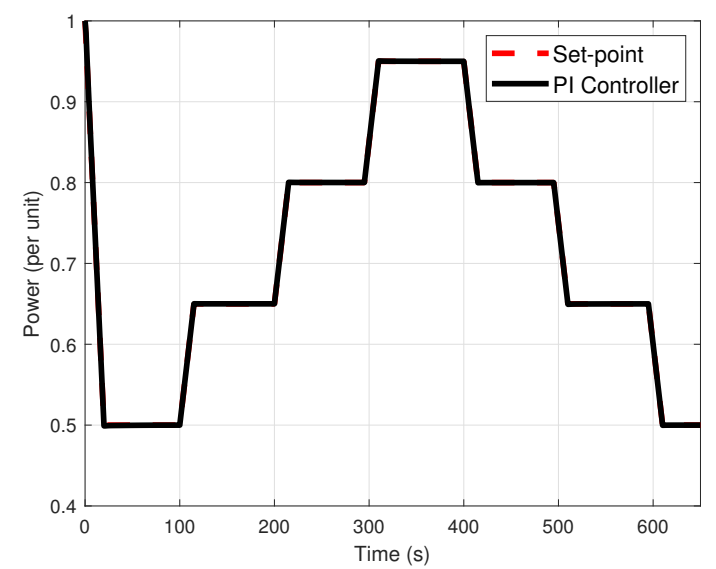

Fig. 4: Variation of the power for normal mode.

control signal (reactivity) as well as the rate of change of reactivity are shown in Fig. 5 and Fig. 6, respectively. In this paper, it is assumed that the reactivity is constrained by $-0.01<\mathrm{u}<0.01$.

In addition to the normal mode, a simulation is performed in the presence of faults in order to evaluate the faulttolerance of the proposed controller. The performance of the controller in case of faults in the actuator and sensor power is shown in Fig. 7. The actuator-based fault can be described as a signal with a period of $5 \mathrm{~s}$ and a magnitude of 7.10-3. The sensor-based fault is the same signal type with a magnitude of 0.016 . It can be observed a variation in the power when the faults are introduced but the controller is able to track the set-point. As for the fault introduced in the actuator, the controller is able to track back the set-point after a period of $8 \mathrm{~s}$ while for the fault in the sensor it needs $5 \mathrm{~s}$ for the correction to be made. However, it can be noticed that the variation of the control signal caused by the faults is important. The control signal is indeed facing an increase of $6.10-3$ at 250 s for only $1 \mathrm{~s}$ in the presence of the fault in the actuator.

\section{CONCLUSIONS}

A FBL strategy based on a DNN has been studied for a PWR nuclear reactor. The DNN was previously trained 


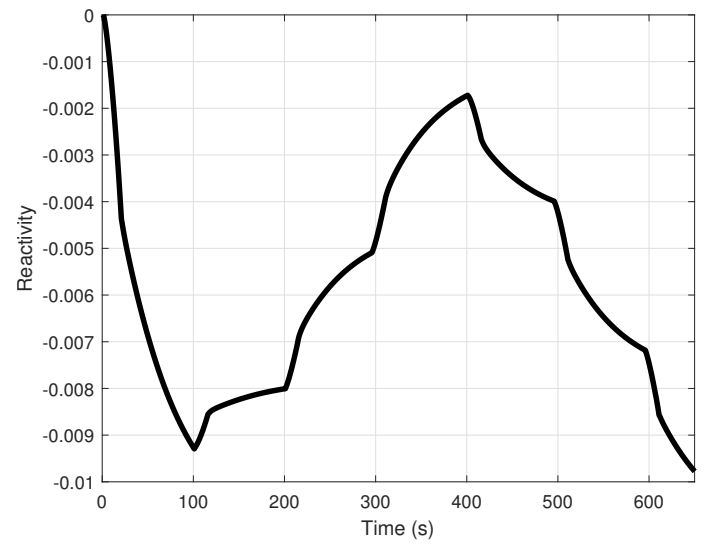

Fig. 5: Variation of the reactivity (control signal) for normal mode.

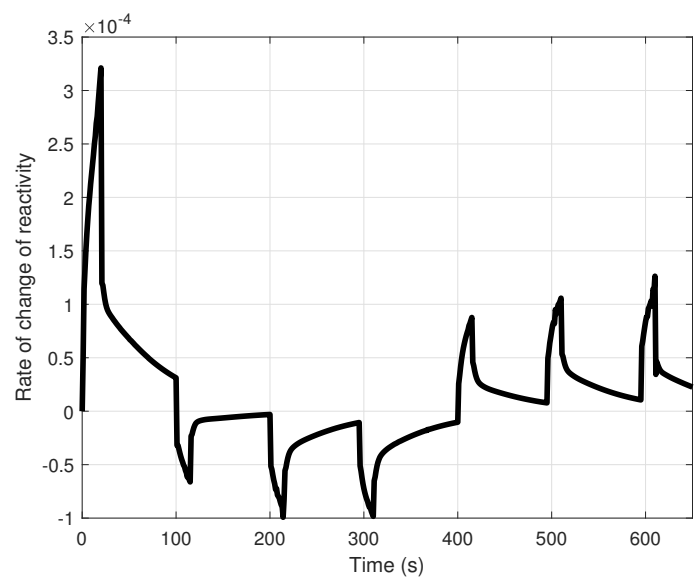

Fig. 6: Variation of rate of change of reactivity for nornal mode.

offline using the Quasi-Newton algorithm.The simulation results demonstrate the effectiveness and the good performance of the proposed controller for load-following. The proposed control method is able to track the desired power with a good precision. Moreover, a simulation is performed with the presence of a fault in the actuator and in the sensor. The control strategy is proved to be able to deal with the perturbations. However, the level of control effort is found to be important in the presence of faults.

\section{ACKNOWLEDGEMENT}

The work presented in this paper has been financially supported under grants EP/R021961/1, EP/R022062/1, and EP/M018717/1 from the Engineering and Physical Sciences Research Council.

\section{REFERENCES}

[1] S. Bennett, "The past of pid controllers," Annual Reviews in Control, vol. 25, pp. 43 - 53, 2001.

[2] K. H. Ang, G. Chong, and Y. Li, "Pid control system analysis, design, and technology," IEEE transactions on control systems technology, vol. 13 , no. 4 , pp. 559-576, 2005.

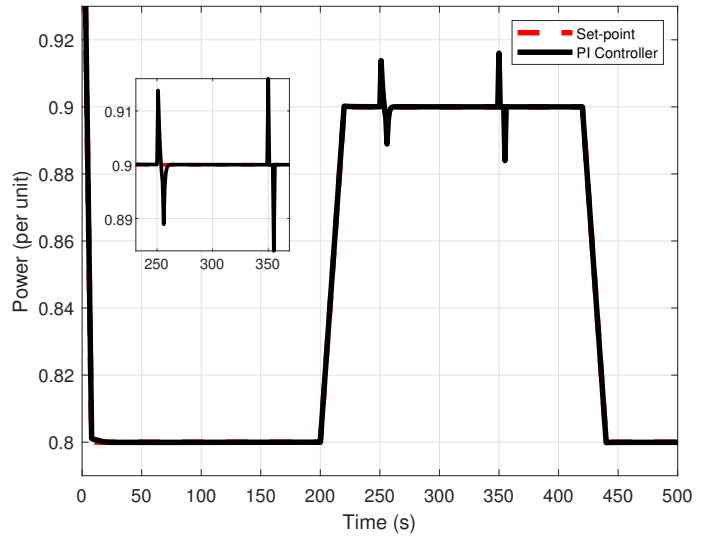

(a) Power variation.

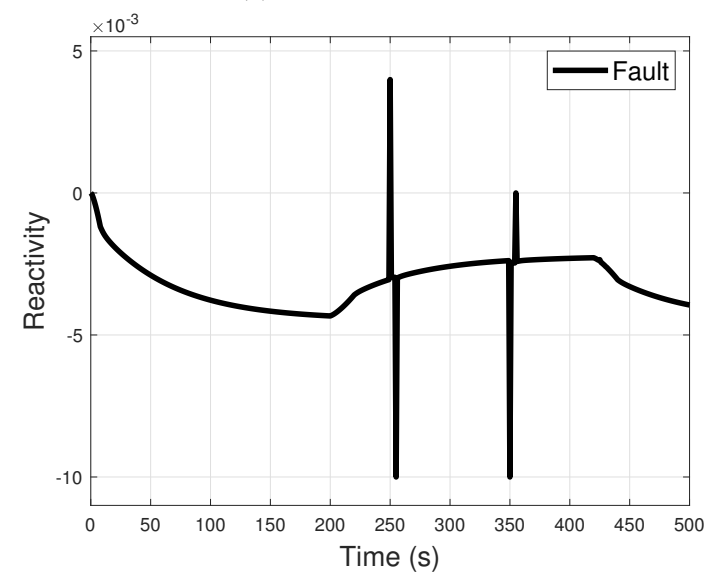

(b) Control signal variation.

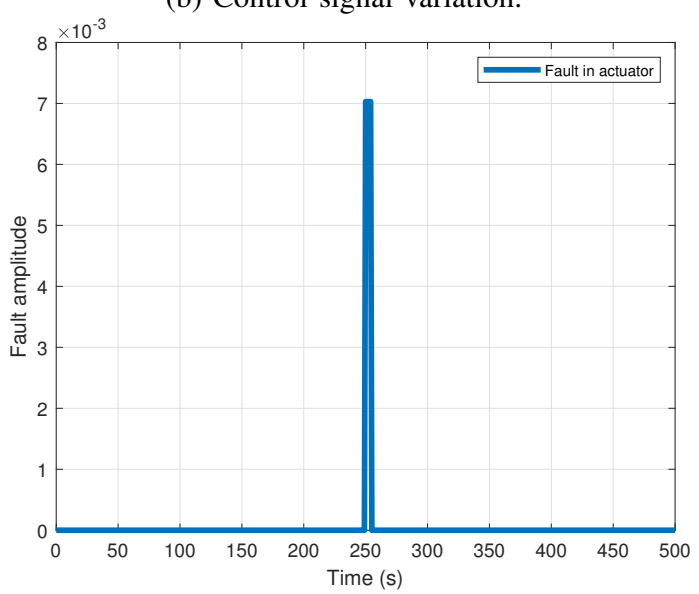

(c) Disturbance applied in actuator.

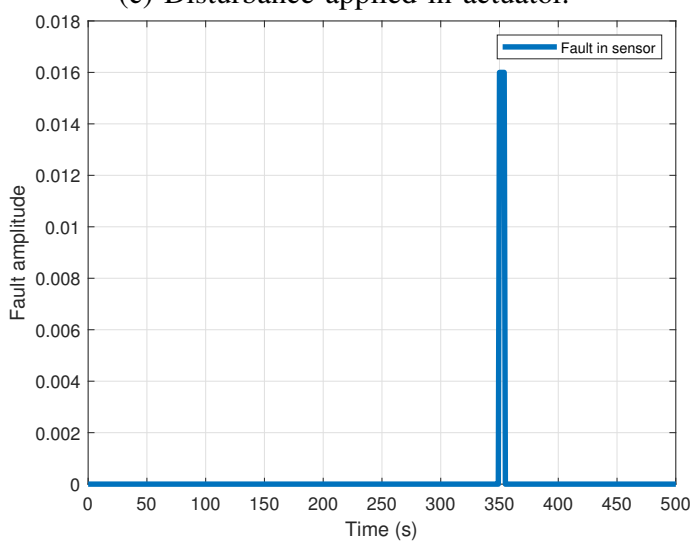

(d) Disturbance applied in sensor.

Fig. 7: Load tracking performance of proposed control in the presence of faults. 
[3] S. M. H. Mousakazemi, N. Ayoobian, and G. R. Ansarifar, "Control of the pressurized water nuclear reactors power using optimized proportional-integral-derivative controller with particle swarm optimization algorithm," Nuclear Engineering and Technology, vol. 50, no. 6, pp. $877-885,2018$.

[4] M. N. Khajavi, M. B. Menhaj, and A. A. Suratgar, "Fuzzy adaptive robust optimal controller to increase load following capability of nuclear reactors," in PowerCon 2000. 2000 International Conference on Power System Technology. Proceedings (Cat. No.00EX409), vol. 1, 2000, pp. 115-120 vol.1.

[5] H. L. Akin and V. Altin, "Rule-based fuzzy logic controller for a pwrtype nuclear power plant," IEEE Transactions on Nuclear Science, vol. 38, no. 2, pp. 883-890, 1991.

[6] S. S. Khorramabadi, M. Boroushaki, and C. Lucas, "Emotional learning based intelligent controller for a pwr nuclear reactor core during load following operation," Annals of Nuclear Energy, vol. 35, no. 11, pp. $2051-2058,2008$.

[7] H. Eliasi, M. Menhaj, and H. Davilu, "Robust nonlinear model predictive control for nuclear power plants in load following operations with bounded xenon oscillations," Nuclear Engineering and Design, vol. 241 , no. 2 , pp. $533-543,2011$.

[8] Z. Alavi, M. Menhaj, and H. Eliasi, "Model reference adaptive control of a nuclear reactor," in 2009 International Conference on Mechatronics and Automation. IEEE, 2009, pp. 735-740.

[9] M. Eom, D. Chwa, and D. Baang, "Robust disturbance observer-based feedback linearization control for a research reactor considering a power change rate constraint," IEEE Transactions on Nuclear Science, vol. 62, no. 3, pp. 1301-1312, 2015.

[10] G. Ansarifar et al., "Robust feedback-linearization control for axial power distribution in pressurized water reactors during load-following operation," Nuclear Engineering and Technology, vol. 50, no. 1, pp. 97-106, 2018.

[11] A. Naimi, J. Deng, A. Abdulrahman, V. Vajpayee, B. Victor, and N. Bausch, "Dynamic neural network-based system identification of a pressurized water reactor," 2020.

[12] F. Garces, V. Becerra, C. Kambhampati, and K. Warwick, Strategies for feedback linearisation: a dynamic neural network approach, 2002.

[13] A. Yeşildirek and F. L. Lewis, "Feedback linearization using neural networks," Automatica, vol. 31, no. 11, pp. 1659-1664, 1995.

[14] S. He, K. Relf, and R. Unbehauen, "A neural approach for control of nonlinear systems with feedback linearization," IEEE Transactions on Neural Networks, vol. 9, no. 6, pp. 1409-1421, 1998.

[15] J. R. Alique, R. E. Haber, and A. Alique, "Feedback linearization using neural networks: Application to an electromechanical process," in International Work-Conference on Artificial Neural Networks. Springer, 2003, pp. 758-765.

[16] N. Ranchod and J. O. Pedro, "Dynamic neural network-based feedback linearization control of antilock braking systems incorporated with active suspensions," in 2019 12th Asian Control Conference (ASCC). IEEE, 2019, pp. 331-336.

[17] J. O. Pedro, M. Dangor, O. A. Dahunsi, and M. M. Ali, "Dynamic neural network-based feedback linearization control of full-car suspensions using pso," Applied Soft Computing, vol. 70, pp. 723 - 736, 2018.

[18] J. Deng, V. M. Becerra, and R. Stobart, "Predictive control using feedback linearization based on dynamic neural models," in 2007 IEEE International Conference on Systems, Man and Cybernetics, 2007, pp. 2716-2721.

[19] V. Vajpayee, V. Becerra, N. Bausch, J. Deng, S. Shimjith, and A. J. Arul, "Dynamic modelling, simulation, and control design of a pressurized water-type nuclear power plant," Nuclear Engineering and Design, vol. 370, p. 110901, 2020. 